\title{
SOLUTION OF VISCOUS FLOW IN A RECTANGULAR REGION BY USING THE HYBRID FINITE VOLUME SCHEME
}

\author{
Vusala Ambethkar, Lakshmi Rani Basumatary \\ Department of Mathematics, Faculty of Mathematical Sciences \\ University of Delhi, Delhi-110007, India \\ vambethkar@maths.du.ac.in,vambethkar@gmail.com,Irbasumatary@gmail.com
}

Received: 20 June 2019; Accepted: 3 October 2019

\begin{abstract}
In the present work, a solution to the problem of viscous flow in a rectangular region with two moving parallel walls is obtained by using a hybrid finite volume scheme. The discretized governing equations are solved iteratively, and thereby the flow variables are computed numerically. The results for velocity and pressure in horizontal and vertical directions through the centre of a rectangular region are elucidated. The nature of velocity profiles and pressure for different Reynolds numbers in the horizontal and vertical directions through the geometric centre was analyzed with the help of pictorial representations. The present results are compared with the available benchmark results and we have found that they are not in disagreement.
\end{abstract}

MSC 2010: 76D05, $76 M 12$

Keywords: components of velocity, pressure, moving parallel walls, rectangular region, hybrid finite volume scheme

\section{Introduction}

The mathematical model for analysing the viscous flow in a rectangular region with two moving parallel walls occurs in industrial applications, such as chemical etching or film coating industries. There have been numerous investigations carried out over the last decade regarding viscous flow in a square cavity. However, we have discovered few studies on a viscous flow in a rectangular cavity with a moving top wall.

A time-marching numerical method for computation of correct velocity and pressure fields of a general fluid flow problem was studied by Patankar and Spalding [1]. A coupled strongly implicit multigrid method based on the stream function-vorticity formulation was suggested by Ghia et al. [2] for finding the solutions of a twodimensional incompressible flow in a square cavity. A capable solution strategy for solution of steady incompressible flow using the automatic adaptive refinement method was investigated by Thompso and Ferziger [3]. Bruneau and Jouron [4] have investigated an efficient scheme for solving steady incompressible Navier-Stokes 
equations with the help of the implicit relaxation technique coupled using a simplified FMG-FAS algorithm. Liakos [5] proposed a two level method and its algorithm for the solution of nonlinear system of equations for viscous flow. The high order accurate compact finite volume method for steady and unsteady flows was investigated by Piller and Stalio [6]. A compact fourth-order finite volume method for the solutions of Navier-Stokes equations on a staggered grid was proposed by Hokpunna and Manhart [7].

From the review of literature and to the best of our knowledge, we observed that, flow in a rectangular region with two moving parallel walls has not yet been attempted by the researchers using hybrid finite volume scheme. Therefore, our goal here is to fill this gap. Hence we are motivated to perform this current study.

The motive of this current study is to find the solution of viscous flow in a rectangular region with two moving parallel walls using a hybrid scheme [8]. The quasilinear partial differential equations are discretized using this scheme. The semiimplicit method for pressure linked equations is an algorithm that is based on an iterative method of solution is used in this current study. Consequently, flow variables are computed numerically and explicated in the horizontal and vertical directions through the centre of the region. Code verification of the present problem with the benchmark solutions is carried out.

\section{Mathematical formulation}

\subsection{Configuration description}

The configuration of flow in a rectangular region ABCD of length $L$ and breadth $H$ with suitable boundary conditions is presented in Figure 1. Since we have assumed that the boundaries $\mathrm{AD}$ and $\mathrm{BC}$ are free boundary walls, slip wall boundary conditions for velocity $u=10$ and -10 are defined on the boundary walls AD and $\mathrm{BC}$ respectively [9]. Consequently, the flow is defined to be normal to the boundary

walls DC and AB. Hence, the normal velocity $\frac{\partial v}{\partial x}$ defined to be zero on the boundary walls $\mathrm{DC}$ and $\mathrm{AB}$. At all corner points of the region, velocity components $(u, v)$ and pressure $P$ assumed to be zero, i.e., it must be noted here that, excluding the corner points, the boundary conditions for velocity components on the boundary walls of the region $\mathrm{ABCD}$ are defined as mentioned above.

\subsection{Governing equations}

The present model constitutes a 2-D steady, incompressible, viscous flow in a two moving parallel plates of a rectangular region [10]. The governing equations to this model in dimensional form are given as follows:

Continuity equation: $\quad \frac{\partial U}{\partial X}+\frac{\partial V}{\partial Y}=0$ 


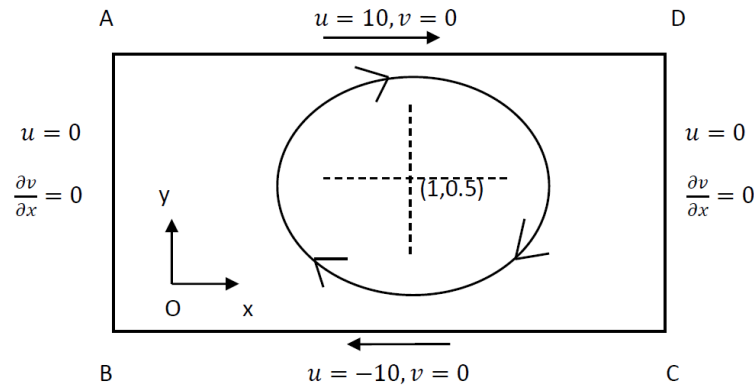

Fig. 1. Design of the problem

$X$-momentum equation: $U \frac{\partial U}{\partial X}+V \frac{\partial U}{\partial Y}=-\frac{1}{\rho} \frac{\partial p}{\partial X}+v\left(\frac{\partial^{2} U}{\partial X^{2}}+\frac{\partial^{2} U}{\partial Y^{2}}\right)$,

$Y$-momentum equation: $U \frac{\partial V}{\partial X}+V \frac{\partial V}{\partial Y}=-\frac{1}{\rho} \frac{\partial p}{\partial Y}+v\left(\frac{\partial^{2} V}{\partial X^{2}}+\frac{\partial^{2} V}{\partial Y^{2}}\right)$.

where $U, V, p, \rho$ and $v$ are the velocity components along the $X$ and $Y$-axis, pressure, density and kinematic viscosity respectively. We define the following dimensionless variables:

$$
(x, y)=\frac{(X, Y)}{L},(u, v)=\frac{(U, V)}{u_{0}}, P=\frac{p}{\rho u_{0}^{2}}, \quad P r=\frac{v}{\alpha} .
$$

The above equations (1)-(3) are subject to the boundary conditions [11] in the dimensional form:

on $\mathrm{AB}: \quad$ at $X=0, U=0, \frac{\partial V}{\partial X}=0 ; \quad$ on DC: $\quad$ at $X=L, U=0, \frac{\partial V}{\partial X}=0$; on $\mathrm{BC}$ : $\quad$ at $Y=0, U=-10 u_{0}, V=0 ; \quad$ on $\mathrm{AD}: \quad$ at $Y=H, U=10 u_{0}, V=0$.

The dimensionless forms of the governing equations (1)-(3) reduce to

Continuity equation: $\quad \frac{\partial u}{\partial x}+\frac{\partial v}{\partial y}=0$,

$x$-momentum equation: $\quad u \frac{\partial u}{\partial x}+v \frac{\partial u}{\partial y}=-\frac{\partial P}{\partial x}+\frac{1}{R e}\left(\frac{\partial^{2} u}{\partial x^{2}}+\frac{\partial^{2} u}{\partial y^{2}}\right)$,

$y$-momentum equation: $\quad u \frac{\partial v}{\partial x}+v \frac{\partial v}{\partial y}=-\frac{\partial P}{\partial y}+\frac{1}{R e}\left(\frac{\partial^{2} v}{\partial x^{2}}+\frac{\partial^{2} v}{\partial y^{2}}\right)$.

where the non-dimensionless variables $u, v, P$ and $R e$ are the velocity components along $x$ and $y$-axis, pressure and Reynolds number respectively. The above equations (5)-(7) are subject to the following boundary conditions in dimensionless form:

$\left.\begin{array}{l}\text { on } \mathrm{AB}: \quad \text { at } x=0, u=0, \frac{\partial v}{\partial x}=0 ; \quad \text { on } \mathrm{DC}: \quad \text { at } x=2, u=0, \frac{\partial v}{\partial x}=0 ; \\ \text { on } \mathrm{BC}: \quad \text { at } y=0, u=-10, v=0 ; \quad \text { on } \mathrm{AD}: \quad \text { at } y=1, u=10, v=0 .\end{array}\right\}$ 


\section{Numerical method}

\subsection{Discretization technique}

The governing equations (5)-(7) are discretized with the help of a hybrid scheme under finite volume set up and obtained the solutions of the flow variables $u, v$ and $P$. The configuration of the staggered grid (Fig. 2) [12, p. 194-196] which is given below helps us to understand and obtain solutions in an iterative manner from descretized equations.

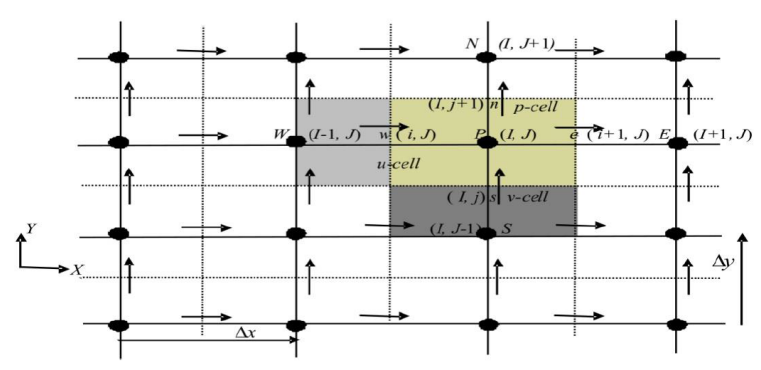

Fig. 2. Staggered grid

\subsection{Discretization of governing equations}

The continuity equation (5) is discretized with the help of scalar control volume as prescribed in Figure 3 [12, p. 202]. The discretized continuity equation at location $(I, J)$ of the scalar control volume is given by

$$
F_{e}-F_{w}+F_{n}-F_{s}=0
$$

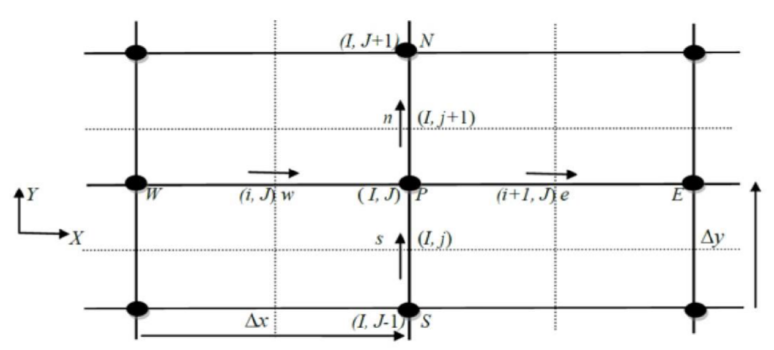

Fig. 3. Scalar control volume (Continuity equation)

The $x$-momentum equation (6) is discretized on $u$-control volume as given in Figure 4 . The discretized $x$-momentum equation at location $(i, J)$ is given by $[12$, p. 197-199]

$$
a_{i, J} u_{i, J}=\sum a_{n b} u_{n b}+\left(P_{I-1, J}-P_{I, J}\right) A_{i, J}
$$


where $A_{i, J}$ is the cell face areas of $u$-control volume. $E, W, N, S$ neighbors involved in the summation are $\sum a_{n b} u_{n b}$ are $(i+1, J),(i-1, J),(i, J+1)$ and $(i, J-1)$. Their locations and prevailing velocities are shown in Figure 4.

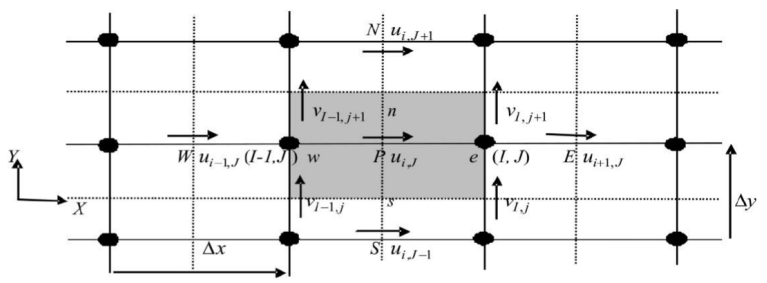

Fig. 4. $u$-control volume

In a hybrid differencing scheme [12, p. 168-169], the coefficients under summation are as follows:

$$
a_{i, J}=a_{i-1, J}+a_{i+1, J}+a_{i, J+1}+a_{i, J-1}+\Delta F
$$

where

$$
\left.\begin{array}{l}
a_{i+1, J}=\max \left[-F_{I, J},\left(D_{I, J}-\frac{F_{I, J}}{2}\right), 0\right], a_{i-1, J}=\max \left[F_{I-1, J},\left(D_{I-1, J}+\frac{F_{I-1, J}}{2}\right), 0\right], \\
a_{i, J+1}=\max \left[-F_{i, j+1},\left(D_{i, j+1}-\frac{F_{i, j+1}}{2}\right), 0\right], a_{i, J-1}=\max \left[F_{i, j},\left(D_{i, j}+\frac{F_{i, j}}{2}\right), 0\right], \\
\Delta F=\left(F_{e}-F_{w}\right)+\left(F_{n}-F_{S}\right)=\left(F_{I, J}-F_{I-1, J}\right)+\left(F_{i, j+1}-F_{i, j}\right)
\end{array}\right\}
$$

where the coefficients contain the combinations of convective flux per unit mass $F$ and diffusive conductance $D$ in $u$-control volume at the cell faces. The values of $F$ and $D$ for each of the interfaces of the $u$-control volume are given as follows:

$$
\left.\begin{array}{l}
F_{e}=F_{I, J}=\frac{F_{i+1, J}+F_{i, J}}{2}=\frac{u_{i+1, J} A_{i+1, J}+u_{i, J} A_{i, J}}{2} \\
F_{w}=F_{I-1, J}=\frac{F_{i, J}+F_{i-1, J}}{2}=\frac{u_{i, J} A_{i, J}+u_{i-1, J} A_{i-1, J}}{2} \\
F_{n}=F_{i, j+1}=\frac{F_{I, j+1}+F_{I-1, j+1}}{2}=\frac{v_{I, j+1} A_{I, j+1}+v_{I-1, j+1} A_{I-1, j+1}}{2} \\
F_{s}=F_{i, j}=\frac{F_{I, j}+F_{I-1, j}}{2}=\frac{v_{I, j} A_{I, j}+v_{I-1, j} A_{I-1, j}}{2} \\
D_{e}=D_{I, J}=\frac{A_{I, J}}{\operatorname{Re} \Delta x}=\frac{1}{\operatorname{Re} \Delta x}\left(\frac{A_{i+1, J}+A_{i, J}}{2}\right), D_{w}=D_{I-1, J}=\frac{A_{I-1, J}}{\operatorname{Re} \Delta x}=\frac{1}{\operatorname{Re} \Delta x}\left(\frac{A_{i, J}+A_{i-1, J}}{2}\right) \\
D_{n}=D_{i, j+1}=\frac{A_{i, j+1}}{\operatorname{Re} \Delta y}=\frac{1}{\operatorname{Re} \Delta y}\left(\frac{A_{I, j+1}+A_{I-1, j+1}}{2}\right), D_{s}=D_{i, j}=\frac{A_{i, j}}{\operatorname{Re\Delta y}}=\frac{1}{\operatorname{Re\Delta y}}\left(\frac{A_{I, j}+A_{I-1, j}}{2}\right)
\end{array}\right\}
$$

Similarly, the $y$-momentum equation (7) is discretized on $v$-control volume and is given in Figure 5. The discretized $y$-momentum equation at $(I, j)$ is given by 
[12, p. 199-200]

$$
a_{I, j} v_{I, j}=\sum a_{n b} v_{n b}+\left(P_{I, J-1}-P_{I, J}\right) A_{I, j}
$$

where the neighbors $E, W, N$ and $S$, involved in the summation $\sum a_{n b} v_{n b}$, are $(I+1, j),(I-1, j),(I, j+1)$ and $(I, j-1)$. Their locations and prevailing velocities are shown in Figure 5.

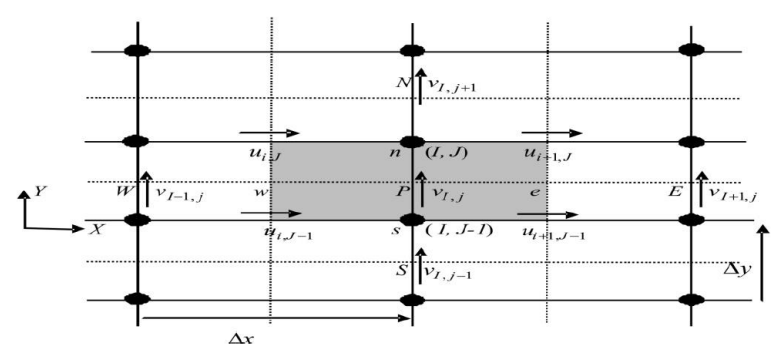

Fig. 5. v-control volume

In the hybrid differencing scheme [12, p. 168-169], the coefficients under summation are defined as follows [13]:

$$
a_{I, j}=a_{I+1, j}+a_{I-1, j}+a_{I, j+1}+a_{I, j-1}+\Delta F
$$

where

$$
\left.\begin{array}{l}
a_{I+1, j}=\max \left[-F_{i+1, j},\left(D_{i+1, j}-\frac{F_{i+1, j}}{2}\right), 0\right], a_{I-1, j}=\max \left[F_{i, j},\left(D_{i, j}+\frac{F_{i, j}}{2}\right), 0\right], \\
a_{I, j+1}=\max \left[-F_{I, J},\left(D_{I, J}-\frac{F_{I, J}}{2}\right), 0\right], a_{I, j-1}=\max \left[F_{I, J-1},\left(D_{I, J-1}+\frac{F_{I, J-1}}{2}\right), 0\right], \\
\Delta F=\left(F_{e}-F_{w}\right)+\left(F_{n}-F_{S}\right)=\left(F_{i+1, j}-F_{i-1, j}\right)+\left(F_{I, J}-F_{I, J-1}\right)
\end{array}\right\}
$$

As per the notations used in $v$-control volume, the values of $F$ and $D$, for each of the faces $e, w, n$ and $s$ reduce to

$$
\begin{aligned}
& F_{e}=F_{i+1, j}=\frac{F_{i+1, J}+F_{i+1, J-1}}{2}=\frac{u_{i+1, J} A_{i+1, J}+u_{i+1, J-1} A_{i+1, J-1}}{2} \\
& F_{w}=F_{i, j}=\frac{F_{i, J}+F_{i, J-1}}{2}=\frac{u_{i, J} A_{i, J-1}+u_{i, J-1} A_{i, J-1}}{2} \\
& F_{n}=F_{I, J}=\frac{F_{I, j}+F_{I, j+1}}{2}=\frac{v_{I, j} A_{I, j+1}+v_{I, j} A_{I, j+1}}{2} \\
& F_{S}=F_{I, J-1}=\frac{F_{I, j-1}+F_{I, j}}{2}=\frac{v_{I, j-1} A_{I, j-1}+v_{I, j} A_{I, j}}{2} \\
& D_{e}=D_{i+1, j}=\frac{A_{i+1, J}}{\operatorname{Re} \Delta x}=\frac{1}{\operatorname{Re} \Delta x}\left(\frac{A_{i+1, J}+A_{i+1, J-1}}{2}\right), D_{w}=D_{i, j}=\frac{A_{i, j}}{\operatorname{Re\Delta x}}=\frac{1}{\operatorname{Re\Delta x}\left(\frac{A_{i, J}+A_{i, J-1}}{2}\right)} \\
& D_{n}=D_{I, J}=\frac{A_{I, J}}{\operatorname{Re\Delta y}}=\frac{1}{\operatorname{Re\Delta y}}\left(\frac{A_{I, j}+A_{I, j+1}}{2}\right), D_{s}=D_{I, J-1}=\frac{A_{I, J-1}}{\operatorname{Re\Delta y}}=\frac{1}{\operatorname{Re\Delta y}}\left(\frac{A_{I, j-1}+A_{I, j}}{2}\right)
\end{aligned}
$$


Finally the pressure correction equation is given by [12, p. 202]

$$
a_{I, J} P_{I, J}^{\prime}=a_{I+1, J} P_{I+1, J}^{\prime}+a_{I-1, J} P_{I-1, J}^{\prime}+a_{I, J+1} P_{I, J+1}^{\prime}+a_{I, J-1} P_{I, J-1}^{\prime}+b_{I, J}^{\prime}
$$

where

$$
a_{I, J}=a_{I+1, J}+a_{I-1, J}+a_{I, J+1}+a_{I, J-1}
$$

and the coefficients are defined as follows:

$$
\left.\begin{array}{l}
a_{I+1, J}=(d A)_{i+1, J}, \quad a_{I-1, J}=(d A)_{i, J}, \\
a_{I, J+1}=(d A)_{I, j+1}, \quad a_{I, J-1}=(d A)_{I, j}, \\
d_{i, J}=\frac{A_{i, J}}{a_{i, J}}, \quad d_{I, j}=\frac{A_{I, j}}{a_{I, j}} \\
b_{I, J}^{\prime}=\left(u^{*} A\right)_{i, J}-\left(u^{*} A\right)_{i+1, J}+\left(v^{*} A\right)_{I, j}-\left(v^{*} A\right)_{I, j+1}
\end{array}\right\}
$$

In the source term $b^{\prime}$, the continuity imbalance is seen due to utilizing the guessed velocity fields.

\subsection{Numerical computations}

Our target is to compute numerical solutions of the flow variables. Since we hereby adopt the well-known algorithm of the semi implicit method for pressure linked equations proposed by Patankar [14]. The steps involved in this algorithm are summarised as follows:

\section{Semi implicit method for pressure linked equations algorithm:}

The semi implicit method for a pressure linked equations algorithm for computing the numerical solutions of fluid flow consists of the following sequence of steps [12, p. 200-204]:

Step 1 Start with guess velocities $u^{*}, v^{*}$ and pressure fields $P^{*}$.

Step 2 Calculate the coefficients in the momentum equations, solve discretized momentum equations.

Step 3 Calculate the coefficients of the pressure equation, solve pressure correction equation.

Step 4 Correct pressure and velocities:

$$
\left.\begin{array}{l}
P_{I, J}=P_{I, J}^{*}+P_{I, J}^{\prime}, \\
u_{i, J}=u_{i, J}^{*}+d_{i, J}\left(P_{I-1, J}^{\prime}-P_{I, J}^{\prime}\right), \\
v_{i, J}=v_{i, J}^{*}+d_{i, J}\left(P_{I, J-1}^{\prime}-P_{I, J}^{\prime}\right) .
\end{array}\right\}
$$

Step 5 Replace the previous intermediate values of pressure and velocity $\left(u^{*}, v^{*}, P^{*}\right)$ with the corrected values $(u, v, P)$, return to Step 2 , and repeat this process until the solution converges. 


\section{Analysis of results}

In this study, we explore steady, incompressible viscous flow in a rectangular region with two parallel moving wall boundaries using a hybrid scheme under finite volume setup. The significance of the present problem is that since we have considered two free parallel boundary walls (top and bottom) of the region, as a result of this, the flow is defined as normal to the boundary walls (left and right). Our target in this study is to explore the nature of the flow due to laminar and turbulence for low, moderate and high Reynolds numbers in all regions such as to the left, right, top and bottom of the centre of the rectangular region. Using the algorithm mentioned in Section 3.3 for the numerical computations, we obtained the numerical solutions of $u$ and $v$-velocities which lie between the boundary conditions defined on the top and bottom walls.

The nature of velocity profiles in $\mathrm{x}$ and $\mathrm{y}$ directions in the rectangular region for different ranges of Reynolds numbers are illuminated in Figure 6. The solutions of $u$-velocity versus different points of $y$ in $0 \leq y \leq 1$ are illustrated in Figure 6a. It depicts the nature of the velocity profiles in a vertical direction ( $u$-velocity profiles) from the bottom to the top wall through the centre of the region. We inferred that, as the Reynolds number increases, the velocity increases uniformly in this region from the bottom wall to the centre. The presence of velocity profiles in this manner is due to the hypothesis that a velocity of $-10 \mathrm{~m} / \mathrm{s}$ is applied on the bottom wall and as a result of this, the flow remains laminar in this range of Reynolds numbers 50 to 400. Whereas for $R e=5000$, we examine the oscillatory behaviour of the velocity profiles while moving from the bottom to top wall. This nature of flow is due to the fact that the flow remains turbulent for a high $R e$ number.

Now in the region from the center up to the top wall, for any $R e$ in the range of 50 to 400 , we discover that velocity profile decreases constantly owing to the velocity of $-10 \mathrm{~m} / \mathrm{s}$ applied on $\mathrm{BC}$ is dissipated while reaching the top wall $\mathrm{AD}$ from the centre. whereas for $R e=5000$, we examine the oscillatory behaviour of the velocity profiles while moving from the centre to the top wall. Furthermore, we also infer that at the top wall, the velocity decreases by increasing the Reynolds number from 50 to 5000. The reason for this is similar to what we have explained above for the bottom wall. Furthermore, it is concluded that at the bottom wall, the velocity increases by increasing the Reynolds number from 50 to 5000.

The solutions of $v$-velocity versus different points of $x$ in $0 \leq x \leq 2$ are illustrated in Figure $6 \mathrm{~b}$. The nature of the velocity profiles along the horizontal line from the left to the right wall through the center of the region is illuminated with the aid of Figure 6b. It depicts that in the region from the left wall $\mathrm{AB}$ to the center, for any Reynolds number $(R e)$ in the range of 50 to 400 , we discover that, the velocity profiles decrease constantly as we are proceeding towards the centre. Furthermore, especially for lower Reynolds numbers $R e=50,100$, we have obtained higher numerical values of $v$-velocity. However, these numerical values are found to be within the allowed limits and hence they are realistic. Hence, the numerical values of $v$-velocity finally 


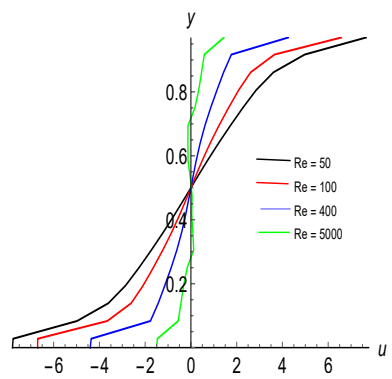

(a)

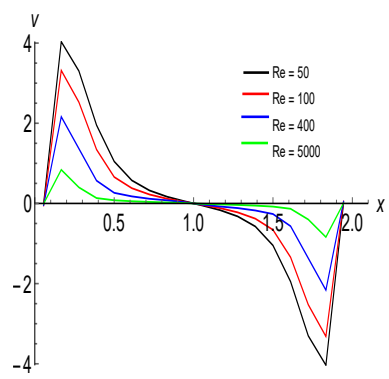

(b)

Fig. 6. (a) $u$-velocity along the vertical line, (b) $v$-velocity along the horizontal line through geometric centre of the rectangular enclosure for $R e=50,100,400,5000$

settles in the realistic limit for a high Reynolds number range rather than the low and the moderate ranges. Furthermore, we can conclude that the numerical values of $v$-velocity for any Reynolds number $(R e)$ greater than $5000(7500,10000,20000, \ldots)$ are almost same as those for $R e=5000$. Now, in the region from the geometric centre until the right wall DC, we have inferred that for any Reynolds number $(R e)$, in the range from 50 to 400 , the $v$-velocity increases constantly. From this, we can conclude that, by increasing the Reynolds number from 50 to 5000, the $v$-velocity increases uniformly from the geometric center towards the right wall. This behaviour is due to the fact that a velocity of $-10 \mathrm{~m} / \mathrm{s}$ applied on bottom wall $\mathrm{BC}$.

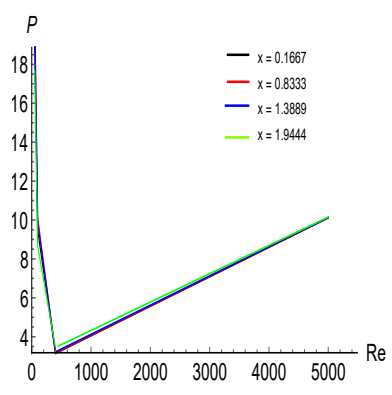

(a)

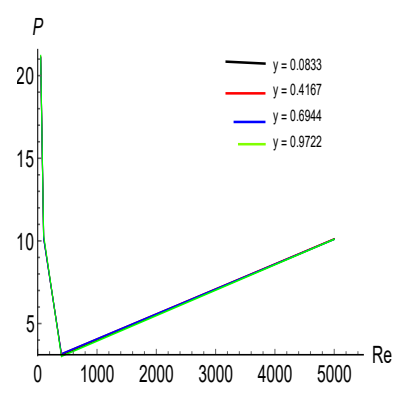

(b)

Fig. 7. Pressure variation for different Reynolds number $R e$ at: a) $y=0.5$, b) $x=1.0$

We have used numerical solutions of pressure at different grid points in drawing Figures 7, which illuminates the variation of pressure profiles along the horizontal and the vertical line through the centre of the rectangular region for different Reynolds numbers in the range of low, moderate and high. Figure 7a depicts that, in the range of low and moderate i.e., when $R e$ is increasing from 50 to 400, the pressure decreases uniformly along the horizontal line of the region through the center. However, from the Reynolds number 400 onwards until 5000 and above, the pressure increases uniformly at all grid points starting near the left wall until the right wall through the centre. The variation of pressure at different points along the verti- 
cal line through the center of the region is illuminated with the help of Figure $7 \mathrm{~b}$. In the range of low and moderate when $R e$ is increasing from 50 to 400 , the pressure decreases uniformly in the direction from the left wall to the right wall of the rectangular enclosure through the geometric center. However, from the Reynolds number 400 onwards until 5000 and above, the pressure increases uniformly at all grid points along the vertical line through the geometric centre.

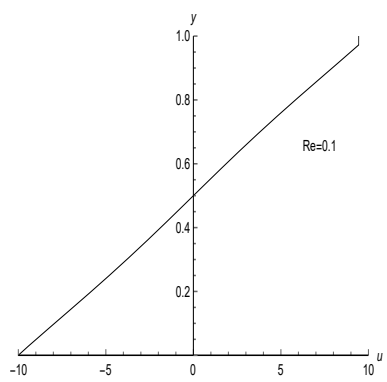

(a)

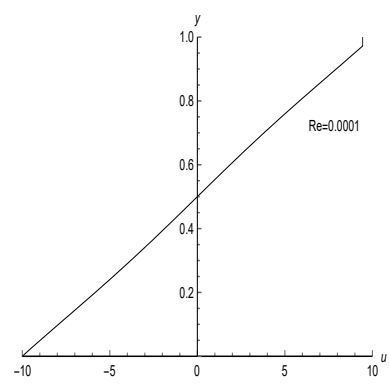

(b)

Fig. 8. $u$-velocity profiles with Reynolds numbers 0.1 (a) and 0.0001 (b)

By considering the importance of the flow behaviour at very low Reynolds numbers $R e<<1$, we have computed the numerical solutions of $u$-velocity at very low Reynolds numbers $R e=0.1$ and 0.0001 and pressure at 0.0001 . These Reynolds numbers correspond to the motion of the tiliate and bacterium in low Reynolds number flow. With the help of these solutions, we have illustrated the velocity profiles along the vertical and pressure profiles along the horizontal direction through the geometric center of the flow.

We inferred from Figures $8 \mathrm{a}$ and $8 \mathrm{~b}$ that for both very low Reynolds numbers 0.1 and 0.0001 , the $u$-velocity increases uniformly in the region from the bottom wall to the center. Now in the region from the center till the top wall, for both the low Reynolds numbers mentioned here, we discovered that the $u$-velocity profiles increase uniformly. The reason for this behaviour of the flow is due to the fact that flow remains mostly laminar at very low Reynolds numbers and the flow does not oscillates.

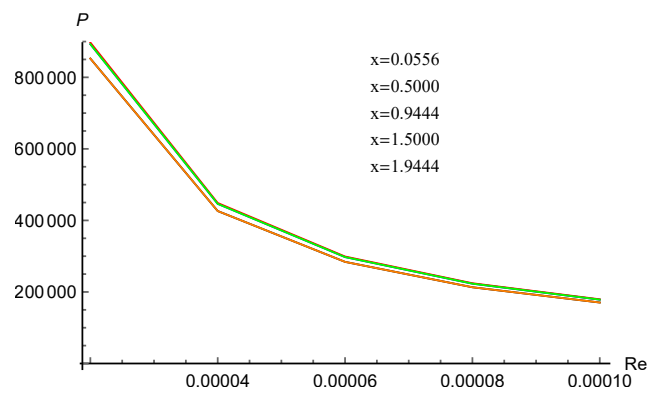

Fig. 9. Pressure profiles for $R e<<1(R e=0.0001)$ 
Since it is very interesting to know pressure profiles in the case of very low Reynolds number flow $(R e<<1)$, we have computed numerical solutions of pressure at different grid points along the horizontal line through the geometric center of the rectangular region in the range of $R e=0$ to 0.0001 . Since motion of bacterium in fluid illustrates the flow at low Reynolds numbers, we have considered an ideal value of $R e=0.0001$ in these computations of pressure at different grid points. The behaviour of pressure profiles at five different grid points is depicted in Figure 9. We have illustrated these pressure profiles for very low Reynolds numbers in the range of $R e=0$ to 0.0001 at five different grid points of $x$ which are shown in this figure and the value of $y$ is at 0.5 . From these pressure profiles, we can conclude that as the Reynolds number increases, the pressure decreases uniformly as we move from the left wall to the right wall through the geometric center of the region.

\section{Code validation}

The velocity profiles along $x$ and $y$-directions for the present problem are computed at the mid-point $(0.5,0.5)$ of the domain using a code written in $\mathrm{C}$. These solutions are computed for Reynolds numbers $R e=100,400,1000,3200,5000$, 7500, 10000 and are compared with benchmark solutions given by Ghia et al. [2]. The results are found to be in good agreement.

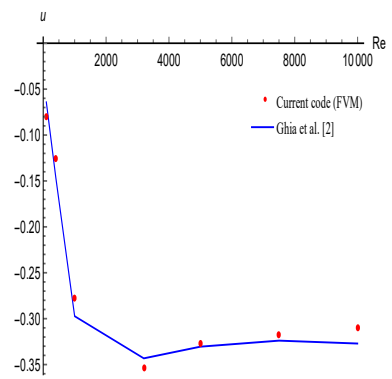

(a)

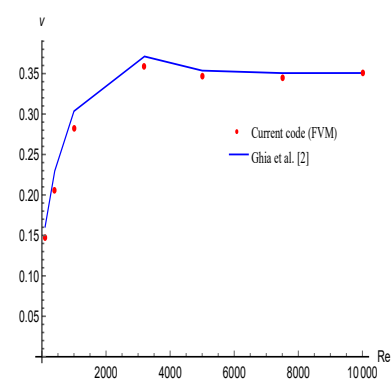

(b)

Fig. 10. Code validation of $u$ (a) and $v$-velocity (b) with benchmark results

\section{Conclusions}

We explore the steady, incompressible viscous flow in a rectangular region with two parallel moving wall boundaries using the hybrid scheme under finite volume setup. Main conclusions of this study are summarized as follows:

- As the Reynolds number increases in the range of 50 to 400 , the velocity increases uniformly in the region from the bottom wall to the centre because a velocity of $-10 \mathrm{~m} / \mathrm{s}$ is applied on the bottom wall $\mathrm{BC}$ and flow remains laminar for low Reynolds numbers as well. 
- In the region from the center to the top wall, increasing the Reynolds number (Re) from 50 to 5000 , the $u$-velocity decreases uniformly because a velocity of $-10 \mathrm{~m} / \mathrm{s}$ applied on $\mathrm{BC}$ has dissipated from the center onwards until the top wall AD. Furthermore, increasing the Reynolds number from 50 to 5000, the $u$-velocity decreases at the top wall. However, it is more at the bottom wall due to the region given above.

- In the region from the left wall $\mathrm{AB}$ until the center, for any Reynolds number $(R e)$ in the range of 50 to 400 , the $v$-velocity decreases uniformly as we are proceeding towards the centre of the region. In the region from the centre until the right wall DC, with increasing Reynolds number from 50 to 5000, the $v$-velocity increases uniformly.

- In the range of low and moderate Reynolds numbers ranging from 50 to 400, the pressure decreases uniformly along the horizontal line of the rectangular region through the centre. However, from $R e=400$ onwards until 5000 and above, the pressure increases uniformly.

- It can be concluded that for low and moderate Reynolds numbers ranging from 50 to 400 , the pressure decreases uniformly along the vertical line through the center. However, when $R e=400$ onwards until 5000 and above, the pressure increases uniformly.

- The numerical solutions for $u$ and $v$-velocity are computed at the mid-point $(0.5,0.5)$ for Reynolds numbers $R e=100,400, \ldots . .10000$ and are compared with benchmark solutions and are found to be in good agreement.

- For both very low Reynolds numbers 0.1 and 0.0001 , the $u$-velocity increases uniformly in the region from the bottom wall to the center and then from center to the top wall of the domain and no oscillatory type of flow is observed in the domain.

- We can conclude that as the Reynolds number increases, the pressure decreases uniformly as we move from the left wall to the right wall through the geometric center of the region.

\section{Acknowledgments}

The second author acknowledges the financial support received from the University Grant Commission, New Delhi under the scheme of the National Fellowship for Higher Education (NFHE) vide letter no. F1-17.1/2016-17/NFST-2015-17-ST-ASS2041, dated April, 2016 to carry out this work. Furthermore, the authors would like to thank the referee for his/her valuable suggestions on the manuscript which helped to improve the quality of the manuscript. 


\section{Nomenclature}

$\begin{array}{ll}\Delta x, \Delta y & \text { grid spacing along } x \text { and } y \text {-axis } \\ u^{*}, v^{*} & \text { guess velocities along horizontal and vertical components } \\ P^{*}, P^{\prime} & \text { guess pressure and presure correction } \\ F_{e}, F_{w}, F_{n}, F_{s} & \begin{array}{l}\text { convective flux per unit mass at east, west, north and south faces } \\ \text { respectively }\end{array} \\ D_{e}, D_{w}, D_{n}, D_{s} & \begin{array}{l}\text { diffusivity conductance at east, west, north and south faces } \\ \text { respectively }\end{array}\end{array}$

\section{Subscripts}

$n b \quad$ neighbouring coordinates

$i, j$ index in tensor form

$e \quad$ finite volume face at $\mathrm{P}$ and $\mathrm{E}$

$w$ finite volume face at $\mathrm{P}$ and $\mathrm{W}$

$n \quad$ finite volume face at $\mathrm{P}$ and $\mathrm{N}$

$s \quad$ finite volume face at $\mathrm{P}$ and $\mathrm{S}$

\section{References}

[1] Patankar, S.V., \& Spalding, D.B. (1972). A calculation procedure for heat, mass and momentum transfer in three-dimensional parabolic flows. Int. J. Heat Mass. Transfer, 15, 1787.

[2] Ghia, U., Ghia, K.N., \& Shin, C.T. (1982). High-Re solutions for incompressible flow using the Navier-Stokes equations and a multigrid method. Journal of Computational Physics, 48 , 387-411.

[3] Thompso, M.C., \& Ferziger, J.H., (1989). An adaptive multigrid technique for the incompressible Navier-Stokes equations. Journal of Computational Physics, 82(1), 94-121.

[4] Bruneau, C.H., \& Jouron, C. (1990). An efficient scheme for solving steady incompressible Navier-Stokes equations. Journal of Computational Physics, 89, 389-413.

[5] Liakos, A. (2001). Discretization of the Navier-Stokes equations with slip boundary conditions. Numerical Methods for Partial Differential Equations, 17, 26-42.

[6] Piller, M., \& Stalio, E. (2004). Finite-volume compact schemes on staggered grids. Journal of Computational Physics, 197, 299-340.

[7] Hokpunna, A., \& Manhart, M. (2010). Compact fourth-order finite volume method for numerical solutions of Navier-Stokes equations on staggered grids. Journal of Computational Physics, 229, 7545-7470.

[8] Chung, T.J. (2010). Computational Fluid Dynamics. Second Edition. Cambridge: Cambridge University Press.

[9] Biringen, S., \& Chow, C.-Y. (2011). An Introduction to Computational Fluid Mechanics by Examples. Hoboken: John Wiley and Sons Inc.

[10] Mostafa Ghiaasiaan, S. (2011). Convective Heat and Mass Transfer. New York: Cambridge University Press.

[11] Bergman, T.L., Lavine, A.S., Incropera, F.P., \& DeWitt, D.P. (2011). Fundamentals of Heat and Mass Transfer. 7th Edition, John Wiley and Sons, USA. 
[12] Versteeg, H.K., \& Malalasekera, W. (2010). An Introduction to Computational Fluid Dynamics: The Finite Volume Method. Pearson Edition, India.

[13] Srivastava, M.K. (2015). A Numerical Study of Viscous Fluid Flow with Heat Transfer Using Marker and Cell(MAC) and Finite Volume Method, Ph.D. Thesis, University of Delhi, India.

[14] Patankar, S.V. (1980). Numerical Heat transfer and Fluid Flow. Washington: Hemisphere/ McGraw-Hill. 